\title{
Experimental Muon Source (EMuS) Target Station Shielding Design for "Baby Scheme"
}

\author{
Nitin Yadav ${ }^{*}$ \\ Institute of High Energy Physics, Beijing \\ E-mail: nitin@ihep.ac.cn
}

\section{Guang Zhao}

Institute of High Energy Physics, Beijing

E-mail: zhaog@ihep.ac.cn

\section{Yuan Ye}

Institute of High Energy Physics, Beijing

E-mail: yuany@ihep.ac.cn

\section{Nikolaos Vassilopoulos}

Institute of High Energy Physics, Beijing

E-mail: vassilopoulos@ihep.ac.cn

The Experimental Muon Source (EMuS) project at China Spallation Neutron Source (CSNS) in Dongguan aims at building a competitive muon source providing both a low energy surface muon beam and a high energy decay muon beam for muon science. Highly polarized positive surface muons are used in muon spin rotation $(\mu \mathrm{SR})$ spectrometers in order to study material properties in condensed matter physics, chemistry and biology. Especially in studying magnetic systems; higher momentum muons can be employed in non-invasive analysis of materials, such as bulk-sensitive elemental analysis with negative muons, or muon radiography techniques. EMuS uses up to $25 \mathrm{~kW}$ proton beam hitting on a graphite target to produce the muon beam with a conventional magnet side collection target station and an advanced forward superconducting solenoid capture target station, so called as "Baby scheme" and "Baseline scheme" respectively. Extremely high radiation produced from the target has to be shielded in order to safeguard the magnet system and the surrounding environment for proper functioning and operation. Therefore, a proper shielding of magnet and environment becomes a key factor of its successful design. We present here the preliminary design and application of hybrid nature of shielding for the EMuS experiment for "Baby scheme".

The 21st international workshop on neutrinos from accelerators (NuFact2019)

August 26 - August 31, 2019

Daegu, Korea

\footnotetext{
* Speaker.

†Alternate email: nitinkantyadav@ gmail.com.
} 


\section{Introduction: Experimental Muon Source Experiment at CSNS}

Powerful proton beams, from accelerators, can be utilized to produce high intensity muon beams by hitting on a suitable target. Muon beams have significant and wide use in fundamental particle physics, nuclear physics and have several multidisciplinary applications. Fundamental research like g-2, rare decay and lepton flavour violating experiments utilize the high intensity muon beams. Highly polarized positive muons, called surface muons that are produced from pions decaying at rest, are used in muon spin rotation $(\mu \mathrm{SR})$ spectrometers in order to study material properties in condensed matter physics, chemistry and biology and particularly used in studying magnetic environments. Higher momentum muons, produced from pions decaying in flight, can be employed in non-invasive analysis of materials such as bulk-sensitive elemental analysis with negative muons or muon radiography techniques.

Since more than thirty years, fundamental researches and multidisciplinary applications continually thrive at muon beam facilities in the world (TRIUMF, FNAL, ISIS/RIKEN-RAL, PSI, J-PARC) [1]. Due to the lack of powerful proton accelerator before, there is no muon source in China. The Experimental Muon Source (EMuS) project [2][3] at China Spallation Neutron Source (CSNS) [4] in Dongguan is planned to develop its first muon source in China. It will be designed to provide both a low energy surface muon beam and a high energy decay muon beam for muon science and especially Muon Spin Rotation ( $\mu \mathrm{SR}$ ) experiments.

EMuS is a part of the strategy plan of CSNS which hosts a $100 \mathrm{~kW}$ and $500 \mathrm{~kW}$ (in its phase I and phase II development plan respectively) proton accelerator with a beam energy of $1.6 \mathrm{GeV}$ and a repetition rate of $25 \mathrm{~Hz}$ [4]. With CSNS phase I completion, the construction of the muon source becomes an important expansion of the CSNS platform in future. A proposed layout of EMuS experiment target station, beam dump and beam transport for surface muon mode and decay muon mode is shown in Figure 1. EMuS consumes proton beam power of 5\% out of the total CSNS proton driver, which translates into $5 \mathrm{~kW}$ and $25 \mathrm{~kW}$ for phase I and phase II respectively at CSNS [2][5] accelerator. This lower power is owed to the fact that the major fraction of the proton beam is sent to the spallation neutron target, without interacting with a muon production target. The total number of hadrons and leptons per second is proportional to proton beam power, which therefore requires specific measures in order to design an appropriate production target and in order to generate muon beam intensities as par with the existing muon facilities across the world.

EMuS is planned to have two target station designs: a conventional magnet sideways (at $90^{\circ}$ ) collection target station and a forward collecting superconducting solenoid capture target station. An extremely high radiation produced in the target stations by the proton beam hitting on target can potentially damage the surrounding magnet system and can disturb the operation at the corresponding facility environment by harmful radiations. Therefore a proper and effective shielding around the target, to protect magnet and environment, becomes a key factor of EMuS successful design. The effect of radiation dose on capture magnets and the prompt and effective doses in the facility should be minimised below a prescribed limit. This is foreseen to be achieved by constructing an appropriate hybrid material shielding structure, containing layers of different materials surrounding the proton-target interaction. As per the requirement, the design should satisfy a limit (as described later) of neutron radiation and integrated dose on the magnetic coils. For example the dose on the wire insulator and epoxy is set to be less than 7 MGy for the lifetime (30 years) 


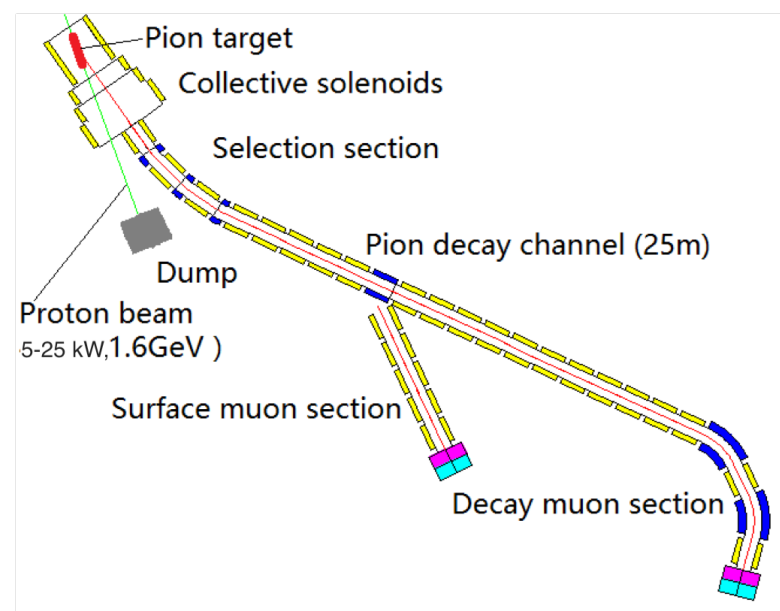

Figure 1: Proposed layout of the EMuS target station with beam transport for "Baseline Scheme".

running of experiments [6].

EMuS is proposed to be designed and constructed in two scheme named as "Baseline scheme" and "Baby scheme". The "Baseline scheme" target station design contains a superconducting capture solenoid with four coils in a step up arrangement with an adiabatic field of $5 \mathrm{~T}$ as proposed in [5][7].The primary protons enter the system and the remaining spent-protons are extracted at 15 degrees between the shields of the fourth coil and matching solenoid. The shield geometry of the capture and matching coils and their positioning is related to the spent-proton forward extraction at a 15 degree angle. A short optimized conical graphite target is located at the centre of the 1st-coil and tilted by 15 degrees in line with proton beam. The matching solenoid is needed to smoothly transport muon and pions downstream to the secondary particle beamline [8]. The capture solenoid is already designed for $5 \mathrm{~kW}$ and is being designed and updated for higher proton beam power of $25 \mathrm{~kW}$. The "Baseline scheme" schematics are shown in Figure 2.

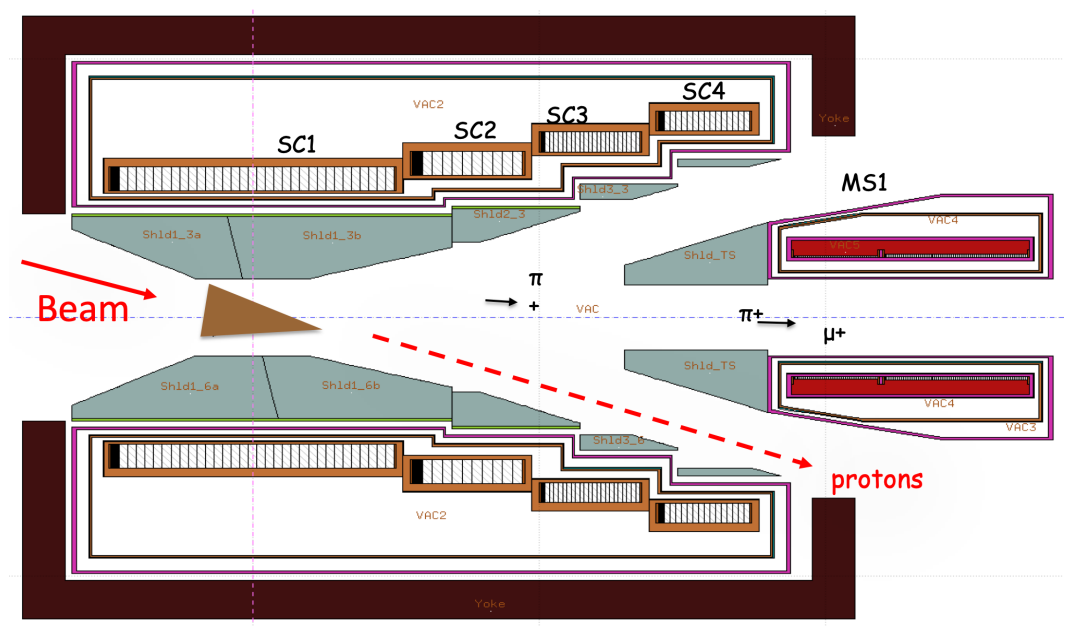

Figure 2: Schematics of the EMuS "Baseline Scheme" target station. Tungsten shields (gray) protecting the four capturing coils (SC1-4) and a matching solenoid (MS1). 
The "Baby scheme" target station consists of a conventional target setup with a small slab of carbon target and sideways (at $90^{\circ}$ ) collection of surface muon by using quadrupole magnet as collectors [3][5] as shown in Figure 3. "Baby scheme" aims to provide a surface muon beam with good momentum resolution and a beam spot size comparable to other muon facilities for $\mu$ SR applications. It will allow the EMuS team gaining valuable experience in the operation of a muon beam line and muon experiment. The valuable experience so gained will highly benefit the realization of a much complex "Baseline scheme" which includes a more complicated target system, solenoidal capture and beam transport with upgraded high intensity proton beam [2][5].

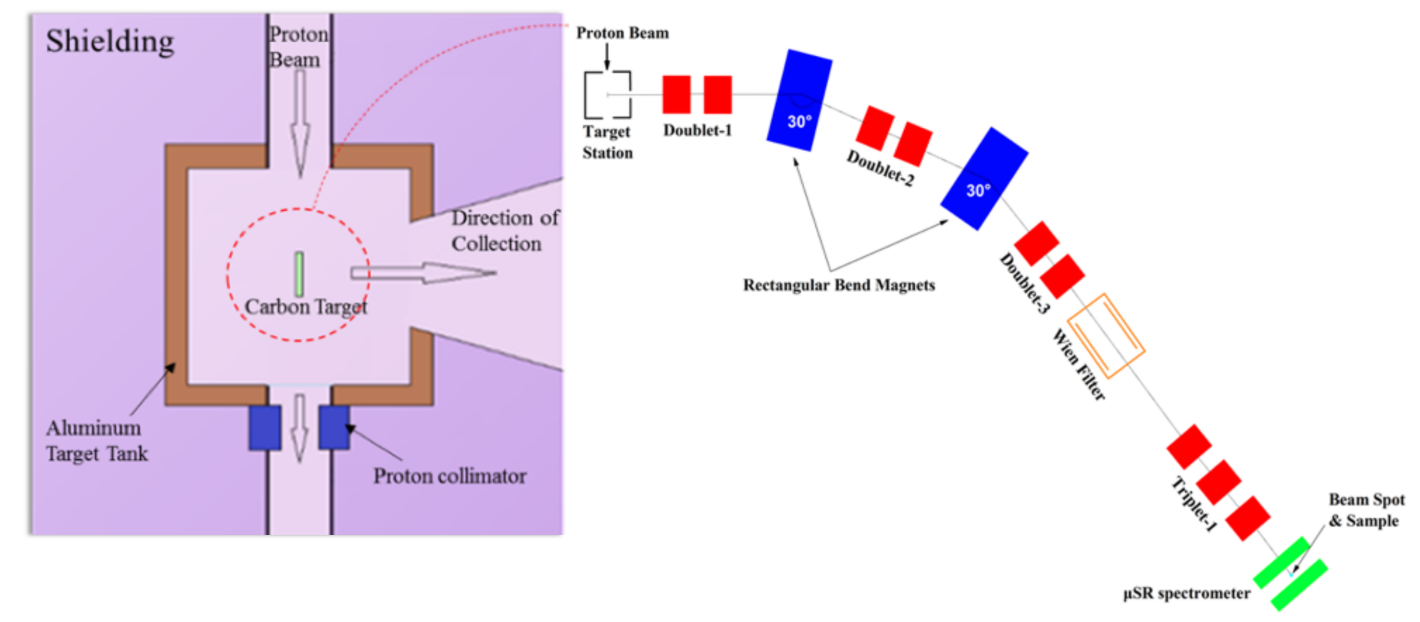

Figure 3: Right side of the figure shows the overview layout of the target station and beam transport of the EMuS "Baby scheme" experiment. On the left is shown the zoomed in figure of the corresponding target station..

\section{EMuS "Baby Scheme" Target Shield Design}

Preliminary studies of dose rates and neutron fluxes are carried out by using FLUKA Monte Carlo simulations [9] for "Baby scheme" at $25 \mathrm{~kW}$. The shield satisfies the criterion of having the maximum dose deposited on the critical components of experiment to be less than prescribed maximum dose limits of $7 \mathrm{MGy}$ for the overall lifetime (30 years) of the experiment. In our simulations we consider a proton beam of energy $1.6 \mathrm{GeV}(25 \mathrm{~kW})$ partially hitting, by centering it on the edge, on a carbon target which is a rectangular slab of dimension- $3 \times 4 \times 8 \mathrm{~cm}^{3}$. An additional safety factor of 2 has also been considered while designing the shield. The shielding design is optimized for a total maximum dose rate parameter at the magnet coils. The shielding is also made as economically viable as possible so as to minimise the overall cost. The resulting shielding is about $60 \mathrm{~cm}$ thick (as measured from the target position) and consists of materials - aluminium, iron and concrete as shown left in Figure 4. With this configuration of hybrid shielding we can achieve the required maximum dose limits, with an additional safety factor of 2 , as can be seen right in Figure 4.

We would also like to mention here about a newly invented alloy which can effectively reduce the total dose rate (by $75-80 \%$ ) due to emanating secondary radiations from the target more effec- 

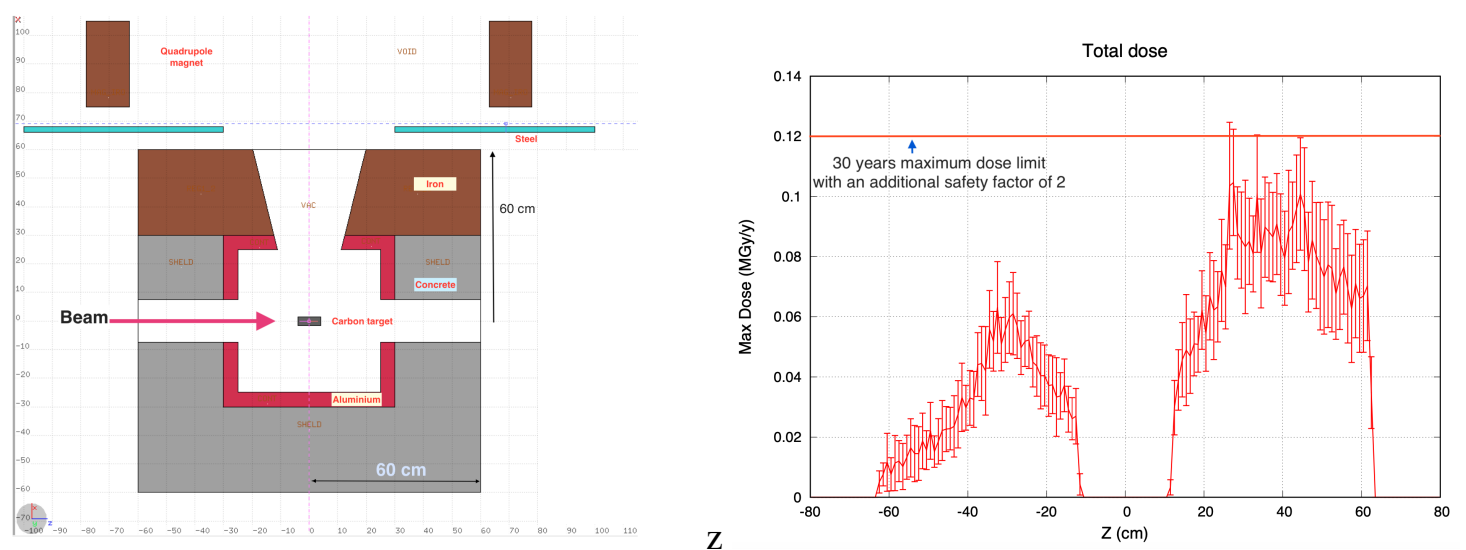

Figure 4: Left: A hybrid shielding solution for "Baby scheme" for $25 \mathrm{~kW}$ proton beam. Right: The maximum total dose rate well below the considered dose of 7 MGy for 30 years with an additional safety factor of 2 .

tively than typical tungsten material shielding. The new alloy comprises of $90 \% \mathrm{~W}$ and a $10 \%$ admixture of Boron, Carbon and Hydrogen.

\section{Conclusions and Acknowledgments}

The preliminary cost effective shield design for EMuS "Baby scheme" has been found out by optimizing the total maximum total dose rate at the magnet coils and results in a hybrid shielding which consists of different materials like aluminium, concrete and iron. The peak dose on critical components after the shielding is well within the considered prescribed dose limit with an additional safety factor of 2 .

The author NY acknowledges Prof J. Tang for his helpful suggestions and discussions. The work has been supported by grants for Postdoctoral Researchers under the CAS President's International Fellowship Initiative (PIFI) for 2019 at Institute of High Energy Physics, Chinese Academy of Sciences Beijing.

\section{References}

[1] https://muonsources.org/muon-centres.html.

[2] J. Tang et al., EMuS Muon Facility and Its Application in the Study of Magnetism, Quantum Beam Sci. 2018, 2(4), 23

[3] H.T. Jing, C. Meng, J.Y. Tang, B.J. Ye, J.L. Sun, Production target and muon collection studies for an experimental muon source at CSNS, Nucl.Instrum.Meth. A684 (2012) 109-116

[4] http://csns.ihep.cas.cn

[5] Experimental Muon Source (EMuS) International Review Conference, CSNS, Dongguan, November 2017

[6] Radiation Hard Coils, A. Zeller et al, 2003, http://supercon.lbl.gov/WAAM 
[7] Nikolaos Vassilopoulos, Zhilong Hou, Ye Yuan, Guang Zhao, EMuS Target Station Studies, J.Phys.Conf.Ser. 874 (2017)

[8] Yu Bao et al., Beamline Design of EMuS - the First Experimental Muon Source in China, J.Phys.Conf.Ser. 1067 (2018)

[9] A. Ferrari, P. Sala, A. Fasso, and J. Ranft, FLUKA: a multi-particle transport code, 2005, CERN-2005-10, INFN/TC 05/11, SLAC-R-773 